\title{
UTILIDAD DE LOS SEGMENTOS CORPORALES PARA LA VALORACIÓN DEL CRECIMIENTO Y EL ESTADO NUTRICIONAL EN NIÑOS Y ADOLESCENTES.
}

\section{USE OF BODY SEGMENTS FOR ASSESS GROWTH AND NUTRITIONAL STATUS IN CHILDREN AND ADOLESCENTS.}

\section{María de las Mercedes Ruiz Brünner ${ }^{1}$, María Elisabeth Cieri' ${ }^{1}$, Antonella Melisa Ferrero², María Dania Zárate $^{2}$, Florencia Bainotti ${ }^{2}$ y Eduardo Cuestas $^{3}$.}

\section{Resumen:}

Introducción: El peso y la talla son datos esenciales para la valoración del crecimiento y estado nutricional en pediatría. La determinación por método directo resulta difícil en pacientes hospitalizados. El objetivo fue analizar la correlación entre el peso y la circunferencia media de brazo (CMB), y entre la talla y la altura talón rodilla (ATR) de niños y adolescentes para su uso en la valoración nutricional y de crecimiento en niños y adolescentes hospitalizados. Población y métodos: Se realizó un estudio observacional, descriptivo y transversal. Se incluyeron, niños, niñas y adolescentes de ambos sexos de 2 y 19 años. Para establecer la correlación se calculó el coeficiente de correlación $r$ y de determinación $R 2$ con una significación de $p<0,05$, graficando la correlación. Para el análisis de los datos se utilizó el software MedCalc V12.5.0.0. Resultados: Se recolectaron datos de 861 sujetos. 484 femeninos (56.2\% IC95\%52,8-59,5), y 377 masculinos (43.8\% IC95\%40,4-47,2) con edades entre 2 y 19 años. Se obtuvo una correlación entre la ATR y la talla de $r=0.98$ para ambos sexos, (R2=0.96 mujeres y $R 2=0.97$ en varones), $p<0.001$. La CMB demostró una correlación con el peso de $r=0.92(R 2=0.76)$ en varones y de $r=0.87$ ( $R 2=0.85)$ en mujeres, ambas con $p<0.001$. Conclusiones: Los segmentos corporales ATR y CMB presentan una alta correlación con la talla y peso, respectivamente. Es por esto que proponemos utilizar los mismos para la valoración del crecimiento y estado nutricional en niños hospitalizados con limitaciones funcionales.

Palabras clave: niño; talla; peso; antropometría.

\section{Abstract:}

Introduction: Weight and height are essential information to assess growth and nutritional status in pediatrics. The determination of them by direct method is difficult in hospitalized patients. The objective was to analyze the correlation between weight and mid arm circumference (MAC), and between height and knee height (KH) of children and teenagers so it can be used to asses growth and nutritional status in hospitalized children. Population and methods: An observational, descriptive and cross-sectional study was carried out. We included children and teenagers of both gender of 2 and 19 years old. To establish the correlation, the correlation coefficient $r$ and determination $R 2$ were calculated with a significance of $p<0.05$, plotting the correlation. The MedCalc V12.5.0.0 software was used to analyze the data. Results: Data from 861 subjects were collected. 484 females (56.2\% Cl95\% 52.8-59.5), and 377 males (43.8\% Cl 95\% 40.4-47.2) aged between 2 and 19 years. A correlation was obtained between the $\mathrm{KH}$ and the height of $r=0.98$ for both sexes, $(R 2=0.96$ females and $R 2=0.97$ in males $), p<0.001$. The MAC showed a correlation with the weight of $r=0.92(R 2=0.76)$ in males and $r=0.87(R 2=0.85)$ in females, both with $p<0.001$. Conclusions: Body segments $\mathrm{KH}$ and MAC have a high correlation with height and weight, respectively. That is why we propose to use them for the assessment of growth and nutritional status in hospitalized children with functional limitations.

Keywords: child; height; weight; anthropometry.

\footnotetext{
1 Lic. en Nutrición, Docente de la Universidad Nacional de Córdoba, Doctoranda en Ciencias de la Salud. Escuela de Nutrición, Facultad de Ciencias Médicas, Universidad Nacional de Córdoba.

2 Lic. en Nutrición, Escuela de Nutrición, Facultad de Ciencias Médicas, Universidad Nacional de Córdoba.

3 Médico Especialista en Pediatría, Magister en Salud Pública, Doctor en Medicina y Cirugía, Profesor Ajunto FCM-UNC, Investigador Clínico de Carrera CONICET. Facultad de Ciencias Médicas, Universidad Nacional de Córdoba.

4 Email de contacto: mercedesruizb@gmail.com
} 


\section{Introducción}

El crecimiento es un aspecto esencial de la salud y un indicador del bienestar de los niños. La valoración nutricional es fundamental para todas las personas, pero principalmente en los niños y adolescentes debido a que el déficit nutricional afecta directamente la velocidad y calidad de su crecimiento y desarrollo. El crecimiento anormal puede indicar una nutrición inadecuada, una enfermedad, o un fracaso para prosperar o sobrevivir, y expresa la vía común en que convergen la nutrición, la salud y el bienestar de un niño(1).

La evaluación nutricional es una herramienta útil para monitorear el crecimiento y la nutrición ${ }^{(2)}$, especialmente en niños hospitalizados, para evaluar la efectividad de las intervenciones médicas en esta población y sus efectos en el crecimiento en pacientes hospitalizados. Entre los métodos usados las medidas directas de peso corporal y altura son las más utilizadas. Estas medidas resultan difíciles o imposibles de obtener en niños que están gravemente enfermos, inmovilizados o tienen deformidades óseas o enfermedad neuromuscular, o en lugares donde no se encuentra disponible el equipo médico necesario. Aunque existen equipos especiales, como balanzas integradas en camas de hospital, estos equipos son costosos y no son accesibles en la gran mayoría de los hospitales y centros de salud de nuestro país. Resulta así necesario utilizar otras estrategias de intervención para poder valorar el crecimiento y el estado nutricional en niños sin la necesidad de la medición del peso o talla de forma directa.

Diversos estudios han evaluado a la ATR y la CMB como medidas indirectas de la talla y el peso respectivamente, comprobando la alta correlación que presentan. Si bien la mayoría de estos estudios se han destinado a la estimación de peso y talla en adultos mayores en países desarrollados ${ }^{(3)}$, existen investigaciones que analizaron esta correlación en la población infantil en otros países ${ }^{(3-11)}$, pero en nuestro conocimiento no existen estudios que hayan analizado esta correlación en el rango etario de 2 a 19 años y en Argentina incluso no existen valores de referencia nacionales publicados de ATR.

Cuando se dificulta obtener la medición de la talla o longitud reclinada se utiliza la medida de segmentos corporales que sirvan para correlacionar con la altura. Las medidas alternativas como ATR, la longitud de la tibia y la longitud del brazo superior se utilizan con frecuencia como medidas indirectas de la talla, ya que por ser huesos largos reflejan una relación entre la talla y el crecimiento lineal de las personas ${ }^{(5,12)}$. A lo largo del tiempo distintos investigadores analizaron diferentes segmentos y muchos han establecido recomendar la medición de la ATR debido a que es fácil de recolectar ya que la rodilla y el talón sirven de topes para la medición, evitando errores en la recolección y proporciona un alto grado de precisión ${ }^{(3-6,13)}$.

Por su parte, la CMB o también llamada circunferencia braquial, es considerada en muchas situaciones como una medida indirecta para valorar el crecimiento normal en niños ${ }^{(5)}$. Permite determinar indirectamente el área grasa, ósea y muscular del brazo, esta última representa una buena indicación de la masa corporal magra y con ello de las reservas proteicas de un sujeto ${ }^{(14)}$. Esta medida, se modifica rápidamente por los cambios en la ingesta calórica y proteica de los sujetos, viéndose reflejado de igual o similar forma que en el peso corporal(15). Se ha demostrado la asociación directa de enfermedades, los cambios bioquímicos, diagnóstico clínico y el estado nutricional con la composición de la parte superior del brazo ${ }^{(7)}$. Esta medición en relación a la edad tiene el propósito de poder diagnosticar y clasificar la desnutrición ${ }^{(14,16-18)}$. Debido a la posible correlación con el peso, resultaría de utilidad el uso de la CMB en la población de niños y adolescentes, como alternativa cuando el peso no pueda ser medido o su medición no sea exacta.

$\mathrm{Si}$ bien existen investigaciones en la población infantil sobre el uso de segmentos corporales para la valoración nutricional o estimación de las medidas directas, existen algunas limitaciones al respecto. En primer lugar, muchas de estas investigaciones se han realizado en poblaciones distintas a la de nuestro país y se basan en datos normativos de poblaciones de muestra que varían ampliamente (por etnia, por capacidad física o por edad), en segundo lugar, los rangos etarios que abarcan muchas veces no son amplios abarcando la población infantil solo en parte (preescolares, escolares o adolescentes). Y por último se han estudiado otros segmentos corporales, como ser el cúbito o la longitud de tibia, que son más difíciles de medir de forma fiable si no se tiene una formación específica, lo cual no resulta práctico en el uso clínico. El objetivo de nuestra investigación fue estudiar el grado de correlación entre la ATR y la talla, y de la CMB y el peso, para así utilizar estos segmentos corporales para evaluar el crecimiento en niños en reemplazo de las medidas directas de talla y peso en quienes estén hospitalizados y no se les pueda valorar directamente, o en hospitales y centros ambulatorios que no disponen del equipamiento necesario. 


\section{Población y Métodos}

Se planeó un estudio observacional, descriptivo de correlación, con recolección prospectiva de los datos. La población de este estudio estuvo conformada por niños y adolescentes sanos de ambos sexos de 2 a 19 años que asistían a controles de salud en el Hospital Privado y el Hospital Pediátrico del Niño Jesús de la Ciudad de Córdoba y a dos instituciones educativas de la provincia de Córdoba, la Escuela Normal Superior Dr. Alejandro Carbó y la Escuela Domingo Zípoli. Los datos fueron recolectados entre Julio 2014 y Julio 2016.

Se realizó un muestreo aleatorio estratificado. La población fue dividida en 17 estratos, de los 2 años y 0 meses a los 18 años y 12 meses. El tamaño muestral se calculó con un potencia de $80 \%$, y una precisión de $1 \%$, en 861 pacientes con 20 niños por estrato de edad (mínimo de 10 por cada estrato según sexo), para un $r$ esperado de al menos 0,90 .

Se incluyeron niños y adolescentes sanos, considerando como sano, a aquellos pacientes clínicamente normales, sin patologías aparentes al momento del examen. Se excluyeron quienes no dieron su consentimiento para participar de la investigación o quienes presentaron desórdenes endócrinos, metabólicos, genéticos y/o anomalías congénitas que afecten o hayan afectado su crecimiento.

\section{Medidas y procedimientos}

\section{Confiabilidad}

Se entrenó a dos investigadores asistentes en el método de medición para cada uno de los cuatro parámetros antropométricos (peso, talla, ATR y CMB). Se realizaron pruebas de fiabilidad (concordancia de al menos $90 \%$ ) inter e intra- observador.

\section{Recolección de peso y talla}

El peso y la estatura se evaluaron con los participantes de pie en ropa interior o ligera y sin calzado de forma independiente: 1) el peso registrado en kilogramos a un decimal registrado a partir de los datos arrojados por la balanza, y 2) para la medición de altura los niños se ubicaron con cabeza, omóplatos, nalgas y talones tocando el tablero de medición, registrando los valores en centímetros y milímetros. Se tuvieron en consideración las recomendaciones de la Sociedad Argentina de Pediatría para la Evaluación del Crecimiento Físico ${ }^{(19)}$.

\section{Medición de Segmentos Corporales}

La CMB se midió en el punto medio entre el acromion y el olecranon. El niño estaba de pie con el brazo doblado $90^{\circ}$ en el codo, y la palma derecha hacia arriba. Se usó una cinta métrica flexible e inelástica regular de fibra de vidrio con una precisión de $1 \mathrm{~mm}$; con un ancho de $6 \mathrm{~mm}$ y una longitud de $2 \mathrm{~m}$. Se tuvo en consideración las recomendaciones para la toma de esta medida establecidas en la Guía de evaluación de crecimiento físico de la Sociedad Argentina de Pediatría ${ }^{(19)}$.

Para medir la ATR el niño estaba sentado en posición vertical con los pies planos en el suelo, con las articulaciones de la rodilla y el tobillo flexionadas en ángulos de $90^{\circ}$. La medida se tomó utilizando un pediómetro portatil marca VARA con una precisión de $1 \mathrm{~mm}$. La ATR se consideró como la distancia desde donde apoya el pie el niño en el extremo fijo del pediómetro, hasta la superficie anterior del muslo, justo proximal a la rótula, donde apoyaba el extremo móvil del instrumento. El eje del pediómetro se mantuvo paralelo a la tibia y una suave presión aplicada en el nivel de la rodilla.

Todas las mediciones se realizaron en ropa ligera sin zapatos y medias. También se recopilaron datos demográficos de sexo y edad decimal del sujeto.

\section{Análisis estadístico}

La normalidad de los datos continuos se probó con la prueba de Kolmogorov-Smirnov y se informaron como media \pm DE. Los datos discretos se expresaron en porcentajes con IC95\%. Para establecer la correlación se calculó el coeficiente de correlación $r$ y de determinación $\mathrm{R}^{2}$, se utilizó un modelo de regresión lineal para identificar la relación entre las variables independientes (circunferencia media del brazo, altura de la rodilla) y las variables dependientes (peso, altura). La significación estadística fue predefinida como un valor de $\mathrm{p}$ 
$<0,05$. Se utilizó el programa MedCalc V12.5.0.0 para el análisis de los datos y para la realización de los gráficos.

Consideraciones éticas: Este estudio fue aprobado por un comité de ética del Hospital Privado de Córdoba contó con la aceptación de los asentimientos de los niños y consentimientos informados escritos de niños mayores de 6 años, adolescentes, padres, madres, tutores y/o cuidadores.

Investigación Financiada por la Dirección de Investigación para la Salud, Ministerio de Salud de la Nación a través de las becas Salud Investiga Dr. Abraam Sonis y la Universidad Nacional de Córdoba, Secretaría de Ciencia y Tecnología, subsidio № 30720150101599CB.

\section{Resultados}

La población estudiada fue de 1.025 sujetos, de los cuales 164 niños fueron excluidos por no cumplir con los criterios de inclusión, ya que se negaron a participar o estuvieron ausentes al momento de la recolección de datos. La muestra quedo conformada por 861 sujetos. De los cuales 484 fueron de sexo femenino $(56.2 \%$ IC95\% 52,8-59,5), y 377 de sexo masculino (43.8\% IC95\% 40,4-47,2) con edades entre 2 y 19 años. El rango de edades decimales para las mujeres fue de 2.375 a 18.923 años de edad, y para los hombres, 2.013 a 18.948 años. Todos los datos se distribuyeron normalmente. Las medias resumen de las edades, peso, altura, CMB y ATR se pueden ver en la Tabla 1.

Tabla 1: Medidas basales de resumen de la muestra total sujetos.

\begin{tabular}{lcccccc}
\hline & $\begin{array}{c}\text { Frecuencia } \\
\mathbf{n}(\%)\end{array}$ & $\begin{array}{c}\text { Edad } \\
(\text { Decimal) } \\
\text { media } \pm \text { DE }\end{array}$ & $\begin{array}{c}\text { Peso }(\text { Kg) } \\
\text { media } \pm \text { DE }\end{array}$ & $\begin{array}{c}\text { Talla }(\mathbf{m}) \\
\text { media } \pm \text { DE }\end{array}$ & $\begin{array}{c}\text { CMB }(\mathbf{c m}) \\
\text { media } \pm \text { DE }\end{array}$ & $\begin{array}{c}\text { ATR(cm) } \\
\text { media } \pm \text { DE }\end{array}$ \\
\hline Sexo & & & & & & \\
Femenino & $484(56,2)$ & $10.6(4.1)$ & $38.0(15.4)$ & $1.38(0.2)$ & $21.0(3.8)$ & $0.43(0.07)$ \\
Masculino & $377(43.8)$ & $10.2(4.4)$ & $38.7(19.2)$ & $1.38(0.2)$ & $21.2(4.6)$ & $0.43(0.09)$ \\
\hline
\end{tabular}

Se determinó la correlación entre la ATR y la talla presentando valores de $r=0.98$ tanto para mujeres como para varones, sin existir diferencia estadísticamente significativa entre sexos. Con un coeficiente de determinación $\mathrm{R}^{2}=0.97$ para mujeres y un $\mathrm{R}^{2}=0.96$ en varones. Todos estos datos fueron estadísticamente significativos con un valor de $p<0.001$. Los gráficos de la correlación se observan en la figura 1 (a y b).

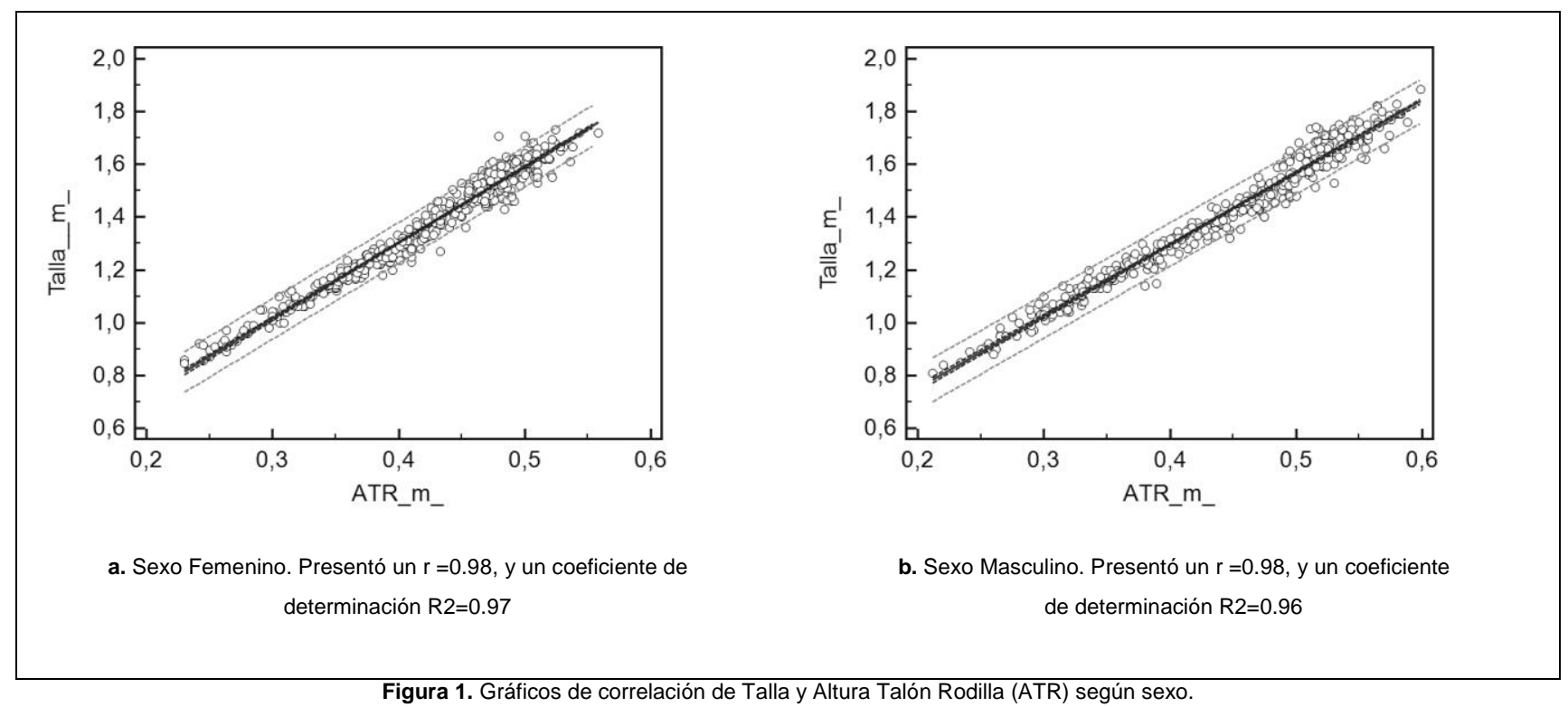

Figura 1. Gráficos de correlación de Talla y Altura Talón Rodilla (ATR) según sexo. 
Por su parte la CMB demostró una correlación con el peso de $r=0.92\left(R^{2}=0.76\right)$ en varones y de $r=0.87$ $\left(R^{2}=0.85\right)$ en mujeres, ambas con una significación de $p<0.001$. Los gráficos de la correlación se observan en la figura 2 ( $a$ y $b)$.

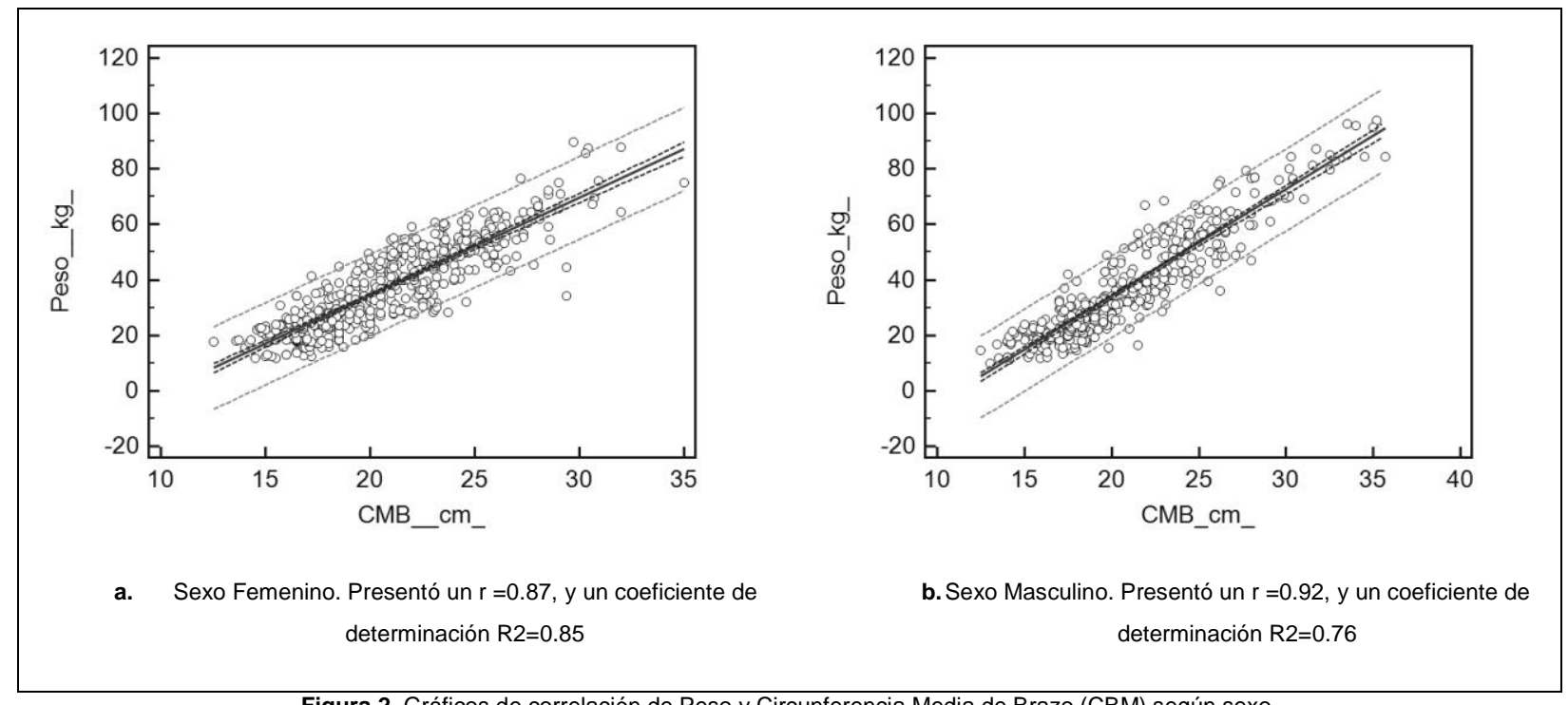

Figura 2. Gráficos de correlación de Peso y Circunferencia Media de Brazo (CBM) según sexo.

\section{Discusión}

Este estudio mostró por primera vez en nuestro conocimiento, que la ATR y la CMB se pueden usar para evaluar en forma indirecta y válida la estatura y el peso en niños y adolescentes desde los 2 a los 19 años de edad.

La ATR presentó en nuestra investigación una correlación muy alta con la estatura en niños de 2 a 19 años de ambos sexos. La alta asociación entre la talla y la ATR demuestra valores similares a los encontrados por Yousafzai et al. ${ }^{(20)}$ en niños de 2 a 6 años en la India y los de Gauld et al. ${ }^{(21)}$ quien trabajó en niños australianos de 5 a 19 años. Al igual que lo planteado por Stevenson ${ }^{(13)}$, se comprobó que dentro de los segmentos que miden huesos largos y se asocian con la talla, la ATR resultó ser la mejor opción ya que los puntos de referencia son más fáciles de palpar, lo que disminuye los errores técnicos. Hemos demostrado que la ATR se puede usar para valorar el crecimiento de la altura en niños cuando no es posible medir la talla en la forma habitual.

La elevada correlación entre el peso y la CMB encontrada en nuestro estudio, muestra que este segmento corporal representa adecuadamente las reservas proteico-energéticas del cuerpo ${ }^{(22)}$, por lo que explica su uso por organizaciones internacionales para evaluar la desnutrición infantil ${ }^{(23,24)}$.

Rabito et al. ${ }^{(5)}$ en un estudio de comparación de métodos de estimación de peso, encontraron que la CMB es una medida que posee un error de menos del 10\%, y puede ser utilizada para valorar le peso en pacientes hospitalizados y/o inmovilizados por solo requerir una cinta métrica y ser una medición práctica, estos hallazgos son coincidentes con los presentados en nuestra investigación. En el Foro del Community-based Management of Acute Malnutrition (CMAM) ${ }^{(25)}$ se recomienda la medición de la CMB, ya que al igual que nuestro estudio, recupera la simplicidad para la recolección de esta medida lo que hace posible que se pueda medir con frecuencia. A su vez en el Foro CMAM se establece que la CMB sirve para evaluar el riesgo a la muerte en niños, esto se debe a que la sobrevivencia está vinculada a la reserva de masa grasa durante la inanición y la reserva de masa muscular para afrontar infecciones, y ambas pueden ser evaluadas mediante la CMB.

Resulta útil poder medir las partes segmentarias del cuerpo como una rutina diaria en la práctica clínica para controlar el crecimiento en niños y adolescentes en los que existan impedimentos para tomar las medidas en forma directa. Si bien los gráficos de crecimiento de las medidas segmentarias, como las existentes de circunferencia del brazo para la edad, pueden facilitar la evaluación del crecimiento en niños y adolescentes, en la medida que no exista acceso a los mismos, como en el caso de la ATR, resulta de utilidad evaluar los cambios de los segmentos corporales del niño a lo largo de su internación o durante su tratamiento médico, ya que estos cambios de ATR y CMB reflejan los cambios en la talla y el peso, respectivamente. 
Entre las limitaciones de este estudio se puede mencionar que las correlaciones se basan en una población representativa mediterránea argentina, y se deberían validar para su aplicación a otras poblaciones con grupos étnicos diferentes. Cabe destacarse que estas correlaciones se derivan para los niños sanos y no son apropiados para su uso en niños con síndromes genéticos o trastornos permanentes que afecten el crecimiento y desarrollo. Se necesitan estudios adicionales en población con discapacidad.

Por otro lado, este estudio presenta fortalezas, ya que es el estudio prospectivo más grande realizado hasta el presente para comprobar el uso de los segmentos corporales a nivel local, realizado con pruebas de fiabilidad inter e intra- observador. La mayoría de los estudios de otros países con muestras de similar tamaño utilizan conjuntos de datos retrospectivos provenientes de bases de datos secundarias, con posibles errores de medición, ya que los datos fueron recolectados por distintos profesionales que no fueron capacitados para realizar las mediciones de igual forma. Esta investigación abre una puerta a nuevos interrogantes: la elevada correlación de los segmentos corporales estudiados con el peso y la talla, permiten pensar que los mismos podrían ser utilizados para la generación de ecuaciones predictivas de estas medidas, la realización de gráficas y tablas de referencia de ATR para la edad para su utilización poblacional en Argentina, y la posibilidad de investigaciones en poblaciones vulnerables con discapacidad que presentan especiales desafíos para la toma de datos antropométricos.

Concluimos que los segmentos corporales ATR y CMB presentan una alta correlación con la talla y peso, respectivamente, en niños y adolescentes. Estas mediciones de 2 a 19 años, son fáciles de efectuar en diferentes contextos clínicos utilizando instrumentos sencillos, de bajo costo, ampliamente distribuidos, por lo que el error de recolección de datos es menor. Es por esto que proponemos utilizar los mismos para la valoración del crecimiento y estado nutricional en niños.

\section{Bibliografía}

1. Lejarraga H. Consideraciones sobre el uso de tablas de crecimiento en la Argentina. Arch Argent Pediatr. 2007;105(6):545-51.

2. Flegal KM, Wei R, Ogden C. Weight-for-stature compared with body mass index-for-age growth charts for the United States from the Centers for Disease Control and Prevention. Am J Clin Nutr [Internet]. 2002 Apr 1 [cited 2016 Feb 18];75(4):761-6. Available from: http://ajcn.nutrition.org/content/75/4/761.long

3. Hogan SE. Knee height as a predictor of recumbent length for individuals with mobility-impaired cerebral palsy. J Am Coll Nutr. 1999;18(2):201-5.

4. Guzmán Hernández C, Reinoza Calderón G, Hernández Hernández $R$ a. Estimación de la estatura a partir de la longitud de pierna medida con cinta métrica. Nutr Hosp. 2005;20(5):358-63.

5. Rabito El, Vannucchi GB, Suen VMM, Neto LLC, Marchini JS. Weight and height prediction of immobilized patients. Rev Nutr. 2006;19(6):655-61.

6. Borba de Amorim R, Coelho Santa Cruz MA, Borges de Souza-Júnior PR, Corrêa da Mota J, González H. C. Medidas de estimación de la estatura aplicadas al índice de masa corporal (IMC) en la evaluación del estado nutricional de adultos mayores. Rev Chil Nutr. 2008;35(1):272-9.

7. Jaswant S, Nitish M. Use of upper-arm anthropometry as measure of body-composition and nutritional assessment in children and adolescents (6-20 years) of Assam, Northeast India. Ethiop J Health Sci [Internet]. 2014;24(3):243$52 . \quad$ Available

http://www.pubmedcentral.nih.gov/articlerender.fcgi?artid=4141228\&tool=pmcentrez\&rendertype=abstract

8. Chowdhury SD, Ghosh T. The upper arm muscle and fat area of Santal children: an evaluation of nutritional status. Acta Paediatr. 2009;98(1):103-6.

9. Myatt M, Duffield A, Seal A, Pasteur F. The effect of body shape on weight-for-height and mid-upper arm circumference based case definitions of acute malnutrition in Ethiopian children. Ann Hum Biol. 2009;36(1):5-20.

10. Hurtado-López EF, Larrosa-Haro A, Vásquez-Garibay EM, Macías-Rosales R, Troyo-Sanromán R, Bojórquez-Ramos MC. Liver function test results predict nutritional status evaluated by arm anthropometric indicators. J Pediatr Gastroenterol Nutr. 2007;45(4):451-7.

11. Abdel-Rahman SM, Paul IM, James LP, Lewandowski A. Evaluation of the Mercy TAPE: Performance Against the Standard for Pediatric Weight Estimation. Ann Emerg Med. 2013;62(4):332-9.

12. Bell KL, Davies PSW. Prediction of height from knee height in children with cerebral palsy and non-disabled children. Ann Hum Biol. 2006;33(4):493-9.

13. Stevenson RD. Use of segmental measures to estimate stature in children with cerebral palsy. Arch Pediatr Adolesc Med. 1995;149(3):658-62.

14. Henríquez-pérez G, Rached-paoli I. Efectividad de la circunferencia del brazo para el despistaje nutricional de niños en atención primaria. Mid-Arm Circumference Effectiveness for the Nutritional Screening of Children in Primary Care . Introducción. An Venez Nutr. 2011;24(1):5-12.

15. Olga Martin A, Rosa A Hernández H. Ecuaciones de predicción del peso corporal para adultos venezolanos. Antropo [Internet]. 2013;29:133-40. Available from: http://www.didac.ehu.es/antropo/29/29-14/Martin.pdf 
16. Lejarraga $\mathrm{H}$, Orfila G. Estándares de peso y estatura para niñas y niños argentinos desde el nacimiento hasta la madurez. Arch Argent Pediatr. 1987;85:209-13.

17. Del Pino M, Bay L, Lejarraga H, Kovalskys I, Pino M. Artículo original Peso y estatura de una muestra nacional de 1.971 adolescentes de 10 a 19 años: las referencias argentinas continúan vigentes. Arch Argent Pediatr. 2005;103(4):323-30.

18. World Health Organization and the United Nations Children's Fund. Child growth standards and the identification of severe acute malnutrition in infants and children. WHO Libr. 2009;1-12.

19. Sociedad Argentina de Pediatría. Guía para la evaluación del crecimiento físico. Tercera Ed. República Argentina: Comité Nacional de Crecimiento y Desarrollo; 2013. 139 p.

20. Yousafzai a K, Filteau SM, Wirz SL, Cole TJ. Comparison of armspan, arm length and tibia length as predictors of actual height of disabled and nondisabled children in Dharavi, Mumbai, India. Eur J Clin Nutr. 2003;57:1230-4.

21. Gauld LM, Kappers J, Carlin JB, Robertson CF. Height prediction from ulna length. Dev Med Child Neurol [Internet]. 2004 Feb 13 [cited 2016 Feb 18];46(7):475-80. Available from: http://doi.wiley.com/10.1111/j.14698749.2004.tb00508.x

22. Ernesto Guevara de la Serna F, del Río Policlínico Manuel González P, Odelkis Alonso Lago D, Daris González Hernández DI, Gladys Abreu Suárez D. Malnutrición proteico-energética en niños menores de 5 años. Rev Cuba Pediatr [Internet]. 2007 [cited 2017 Sep 18];79(2). Available from: http://www.bvs.s/d.cu/revistas/ped/vol79_02_07/ped02207.pdf

23. WHO. WHO child growth standards: head circumference-for-age, arm circumference-for-age, triceps skinfoldfor-age and subscapular skinfold-for-age: methods and development. [Internet]. WHO Library. World Health Organization; 2007. Available from: http://www.who.int/childgrowth/standards/second_set/technical_report_2.pdf

24. Cogill B. Anthropometric Indicators Measurement Guide. Food and nutrition technical asistance proyect. 2001. $1-97 p$.

25. Briend A. Use of MUAC for Severe Acute Malnutrition [Internet]. CMAM Forum. 2012. Available from: http://www.cmamforum.org/Pool/Resources/FAQ-1-Use-of-MUAC-Briend-Eng-June-2012(1).pdf 